\title{
Simulation of multiple scattering in the systems with complicated phase function
}

\author{
E.V. Aksenova*, D.I. Kokorin, V.P. Romanov ${ }^{1}$ \\ Department of Physics, Ul'yanovskaya, 1, Pertodvoretc, 198504 Saint Petersburg, Russia
}

\section{A R T I C L E I N F O}

\section{Article history:}

Received 14 January 2015

Received in revised form

30 June 2015

Accepted 27 July 2015

Available online 4 August 2015

\section{Keywords:}

Multiple scattering

Diffusion of light

Uniaxial systems

\begin{abstract}
A B S T R A C T
We consider simulation of multiple scattering of waves in isotropic and anisotropic media. The focus is on the construction of the phase function interpolation for the single scattering. The procedure is based on the construction of the adaptive partitioning of the angular variables that determine the phase function. The developed interpolation method allows us rather quickly to perform calculations for systems with very complicated phase function. Application of the proposed method is illustrated by calculating the multiple scattering of light in a nematic liquid crystal (NLC) which presents the uniaxial anisotropic system. For this system the grid corresponding to the adaptive partitioning is constructed and the transition to the diffusion regime for the photon distribution is presented.
\end{abstract}

(C) 2015 Elsevier B.V. All rights reserved.

\section{Introduction}

The propagation of light through turbid media is of fundamental interest in a number of areas of optical science including atmospheric optics, biomedicine, ocean optics, suspension optics, liquid crystals optics, communication etc. In most applications, the propagation of light in an isotropic medium is considered. However there are a number of systems such as liquid crystals or biological objects where the anisotropy of the medium is essential. In turbid media, in many cases it is impossible to restrict ourselves to the single scattering of photons and it is necessary to take into account the multiple scattering. There exist various methods which often used to study properties of random medium, e.g. the Monte Carlo method.

Usually the simulation of multiple scattering of photons is based on the knowledge of the phase function of the single scattering. Considerable number of papers is devoted to searching of this function in different cases. So the Mie function is used in atmospheric physics in description of the light scattering by water droplets. The Henyey-Greenstein phase function [1] or its modifications [2] are also frequently used, etc.

For the scattering of light by ice crystals in clouds the phase function of a special form is considered [3-5]. The model which

\footnotetext{
* Corresponding author.

E-mail addresses: aksev@mail.ru (E.V. Aksenova), dmitry@kokorin.org (D.I. Kokorin)

1 Deceased author, February 1, 2015.
}

provides the best agreement with experiment has a rather complicated phase function. This model includes the sum of hexagonal ice aggregates and quasi-spherical ice particles. In the problems of the radiation balance there used the phase function describing the scattering by polydisperse aerosols [6-8].

Similar problems arise in oceanology. Considerable attention is paid to analytical approximations to seawater optical phase functions of scattering [9]. These phase functions vary considerably for different seas and oceans [10,11]. Due to problems with oil pollution of the oceans, considerable attention is paid to the study of the phase functions in oil-in-water emulsion [12,13].

There exist similar problems for diagnostic applications of light in medicine. The solving of this problem also requires the knowledge of the biological object phase functions. Much attention is paid to Monte Carlo modeling of light transport in complex heterogeneous tissues [14,15] and the study of phase functions of blood in the multiple light scattering regime [16-18].

For describing the propagation and scattering of particles there exist program packages such as GEANT4. This program allows us to simulate the propagation and scattering of particles in the real geometry of the experiment. It is particularly useful for the study of elementary particles scattering including the photons. However this package is not designed for studying of multiple scattering of light in media with complicated phase functions. This is due to the fact that it is difficult to set the model of anisotropic scattering which is not embedded in the package.

Another popular approach is based on the simulation of multiple scattering using GPU. This approach reduces the time of calculations significantly using the parallel algorithms. However it 